\title{
Clinical and hystopathological dilemmas in diagnosis of epitheloid hemangioma of maxillae-case report
}

\author{
Mergime Prekazi Loxha ${ }^{1}, Z_{\text {Zana Agani }}{ }^{{ }^{*}}$, Alban Olluri ${ }^{3}$, Fisnik Kurshumliu ${ }^{2}$, Sinan Rusinovci ${ }^{3}$ \\ ${ }^{1}$ University of Prishtina, Faculty of Medicine, Department of Oral and Maxillofacial Surgery \\ ${ }^{2}$ University of Prishtina, Faculty of Medicine, Department of Hysthology \\ ${ }^{3}$ Clinical University Center Of Prishtina, Department of Maxillofacial Surgery
}

\begin{abstract}
Epithelioid hemangioma $(\mathrm{EH})$ is a rare benign tumor involving the blood vessels and surrounding epithelioid cells. Epitheloid hemangioma, also called histiocytoid hemangioma, angiolymcorrect diagnosis of an epithelioid hemangioma $(\mathrm{EH})$, particularly when arising in skeletal locations, because of aggressive clinical characteristics, including multifocal presentation and occasional lymph node involvement. Specifically, its distinction from epithelioid hemangio-endothelioma (EHE) has been controversial. In literature we did not find a case where EH involves maxilla. However, there are few cases reported in which $\mathrm{EH}$ involved maxillary sinus and nasal cavity.

We will present a case of 21 year old male, who came to our Department with a lesion in frontal maxillary region. He underwent surgery four times, wide excision, and with four different histopathology results. The last result was ephiteloid hemangioma with metaplastic bone formation - a very rare benign lesion in oral region, but clinically with very aggressive nature.
\end{abstract} phoid hyperplasia with eosinophilia (ALHE), is a very rare benign Medicine, Department of Oral and tumor in maxillofacial region. There is a controversy in regards to Maxillofacial Surgery

Keywords: Clinical and hystopathological dilemmas, epitheloid hemangioma of maxillae, case report
${ }^{*}$ Correspondence to Author:

Zana Agani

University of Prishtina, Faculty of

How to cite this article:

Mergime Prekazi Loxha, Zana Agani, Alban Olluri, Fisnik Kurshum liu, Sinan Rusinovci.Clinical and hystopathological dilemmas in diag nosis of epitheloid hemangioma of maxillae-case report. International Journal of Dental Research and Reviews, 2018, 1:7

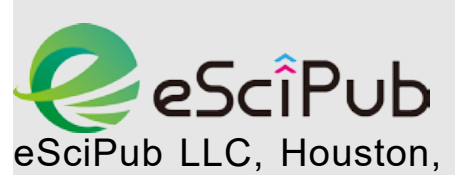

eSciPub LLC, Houston, TX USA. Website: http://escipub.com/ 


\section{Introduction}

An Epithelioid Hemangioma, also known as histiocytoid hemangioma, angiolymphoid hyperplasia with eosinophilia (ALHE) is a rare benign tumor involving the blood vessels and surrounding epithelioid cells. Epitheloid hemangioma may appear in maxillofacial region or in oral cavity. There are limited intraoral cases of $\mathrm{EH}$ and only 16 cases have been reported in the English literature [1]. But sporadic cases are also reported lately in literature. In soft tissues of oral cavity ephiteloid hemangioma may show ulcerative changes with high rate of recurrence. There are rare cases with infiltration of the bone.

\section{Presentation of the case}

In 2016, a 21year old male patient was addmited in our Department for evaluation of anterior alveolar maxillary gingival lesion . Based on the anamnesis, the patient noticed the change about 6 months ago. The main complain was functional and aesthetic disturbance due to the frontal location. Patient denied any history of pain and swelling, exept of occassional bleeding from gingiva corresponding to the same area . During clinical examination we noticed a lesion extending from left central incisor to first left premolar. The mucosa overlying the lession showed reddnes all over the lession, it was painless in palpation , with well circumsised borders and soft consistency.

In $\quad x$-rays

orthopantomography, the radiolucent area between left central incisor and left first premolar was well defined. Vitality of adjent teeth $11,22,23$ and 24 was positive and without any root resorption.

The patient underwent first surgery. The excision of the lesson in toto was done, and wound was packed with jodoform gauze. The specimen was sent for histopathology, and the result was granuloma pyogenicum. The wound healed withou any complication and patient was sent home. After two months patient returned to our clinic with complaine on recurence of the same lession. We underwent the second surgery, again with wide excision and extraction of loosed tooth 22, who was adjected to the lession. The wound was packed with jodoform gauze. The specimen was sent for histopathology, and the result was hemangioma capilaris. Again wound heald and patient was sent home. After three months from last intervention the patient returned again to our Department with the complain of recurence at the same location. We made third operation, with wide excision involving soft tissue and bordering bone tissues involving tooth 23 . The specimen was sent for histopathology, and the result was granuloma gigantocellularis. Laboratory tests :Ca++ total, ca ionisin, phosfor, parathormone, alcaline phosfatase were in normal range.

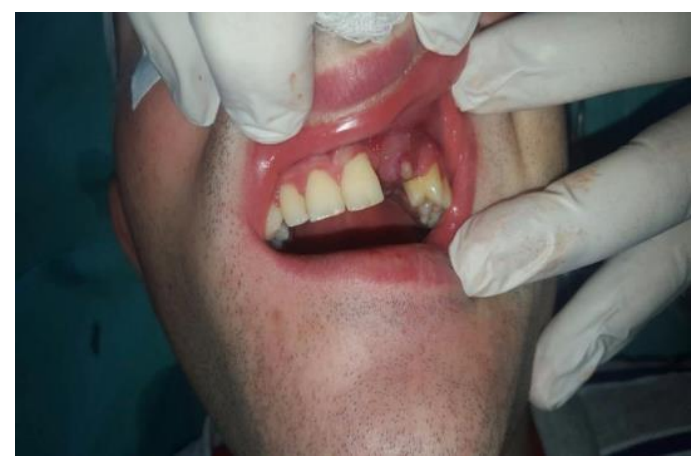

Figure 1. Recurrence of tumor after third operation

Even after third operation the patient returned with recurrence after few weeks (Fig. 1). We decided to underwent the fourth operation. The operation was performed under general 
anesthesia with wide local excision of $10 \mathrm{~mm}$ minimum margin of normal tissue .We were more radical therefore we excised related soft tisssues, anterior maxillary bone together with dens 21 and 24 (Fig.2, Fig. 4) The limits of excision were investigated to ensure clean and safe margins.The overdenture was done to cover the defect, and to ensure function and esthetic (Fig. 3). The speicman was sent for hystopathological examination. The result was plasma cell granuloma. In discussion with pathologist we did imunohistochemistry, used cd marker 31 and 34 and did planing for futher diferentation. Due to our doubts regarding the veracity of HP findings we did sent the specimen in Institute of Pathology University of Ljubljana. The dignosis is further supported by positive FOSB immunohistochemistry. And definitive result was epitheloid hemangioma ulcerated with metaplastic bone formation.(Fig. 5)

The defect is left to heal for second intentionem to observate for recurencies and defect is closed with temporary denture

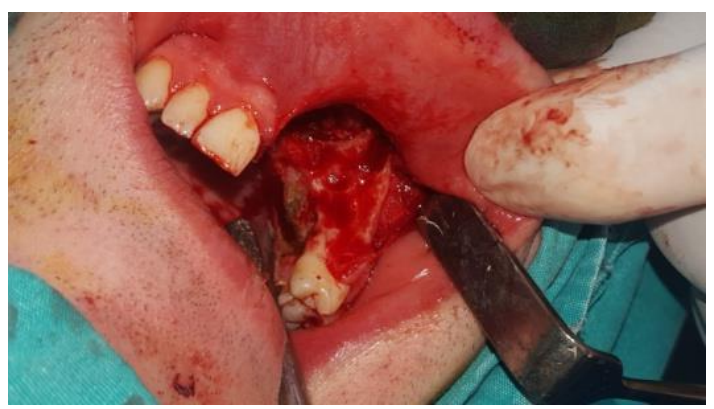

Figure 2. Surgery after fourth operation

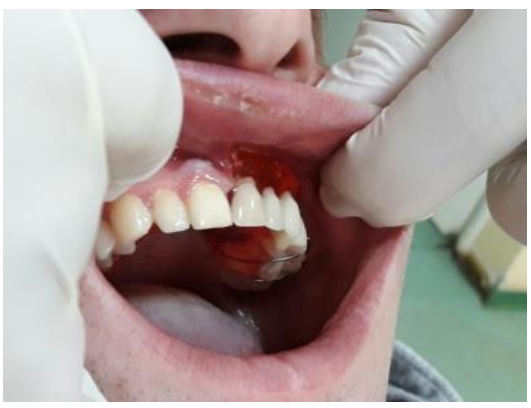

Figure 3. Overdentures after the operation

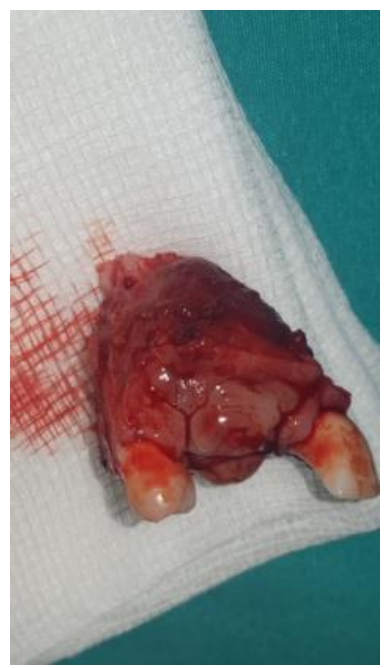

Figure 4. Specimen before final HP result. 


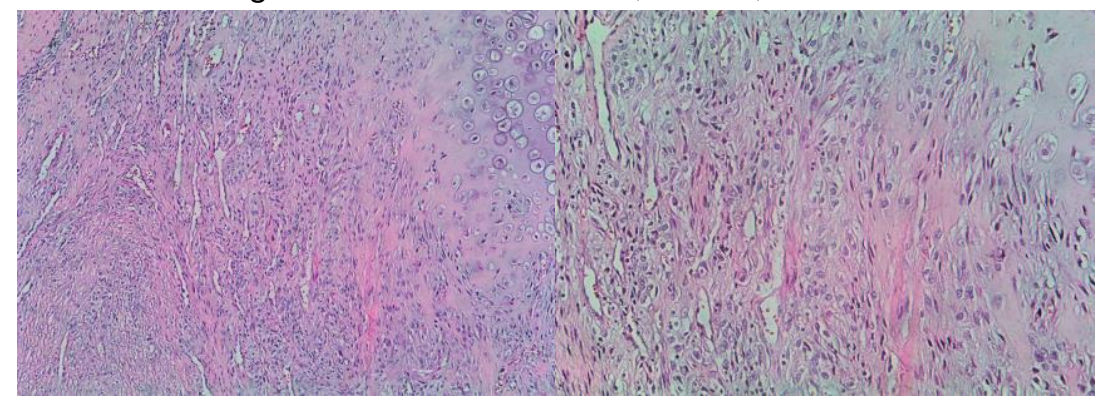

a)

b)

Fig.5. Microscopic view of the specimen showing proliferated blood vessels lined by prominent endothelial cells in the vicinity of nasal cartilage (right hand side of the image) (Hematoxylin \&Eosin stain, a.4x and b. 10x magnification

Further investigationes included ct scan of head and neck, wich resulted negative for regional metastasis.

We proposed consultationes with oncologist for eventual radiotherapy. The patient was in doubt for our methodology of treatment, and disappeared for 3 months. In the mean time he went in a private clinic for consultations where they used laser therapy (Fig. 6). After 3 months that he came to our department with recurrence of the lesion and CT scan showed the infiltration of lateral walls of nasal cavity (Fig .7). Although we proposed to the patient to do another intervention with wide excision- partial maxillectomy, the patient decided to go elsewhere in order to continue his treatment.

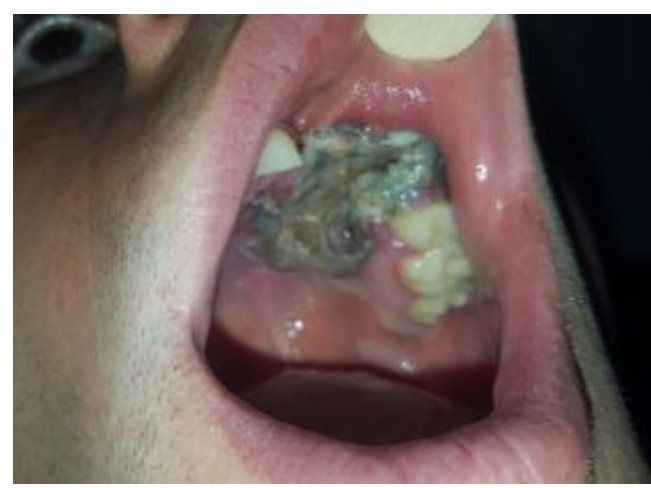

Figure 6. Lesion after laser therapy

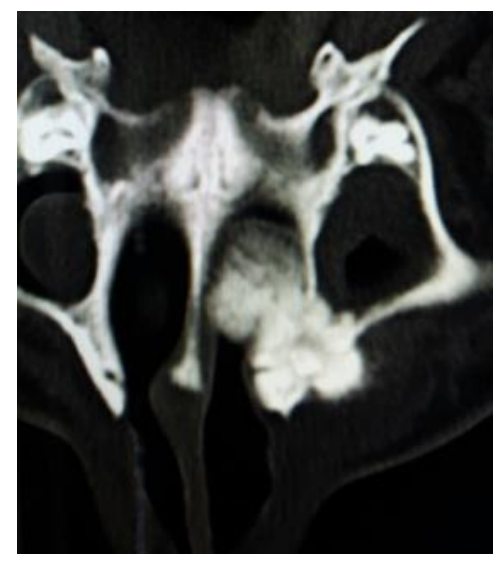

Figure 7. CT scan which shows infiltration of lateral wall of nasal cavity. 


\section{Discussion}

An Epithelioid Hemangioma is a rare, benign tumor involving the blood vessels and surrounding epithelioid cells. Epitheloid hemangioma also called histiocytoid hemangioma, angiolymphoid hyperplasia with eosinophilia (ALHE) may appear as tumor in maxillofacial region- in oral cavity. There are only limited intraoral cases of $\mathrm{EH}$ and only 16 cases have been reported in the English literature [1]. The extracutaneous $\mathrm{EH}$ sites which are more commonly affected are bone, salivary gland, and muscular area or extremities. [3] Histologically, EH is composed of well developed, but frequently immature, vascular structures lined by aggregates of epithelioid- or histiocytoid-like endothelial cells that contain abundant eosinophilic cytoplasm. The endothelial cells are usually associated with a prominent inflammatory infiltrate consisting of dispersed lymphocytes, eosinophils, histiocytes and mast cells. [4]

Epithelioid hemangioma in the oral mucosa is a rare disease and often confused with other epithelioid vascular tumours and nonvascular soft tissue tumours. In the literature various terms have been used by different authors to describe this lesion, including angioblastic hyperplasia with eosinophilia (ALHE), nodular angioblastic lymphoid hyperplasia with eosinophilia, lymphofolliculosis, histiocytoid hemangioma pseudopyogenic granuloma, and atypical pyogenic granuloma. [5].There is a controversy in correct diagnosis of an epithelioid hemangioma (EH), particularly when arising in skeletal locations, because of aggressive clinical characteristics, including multifocal presentation and occasional lymph node involvement. Specifically, the distinction from epithelioid hemangioendothelioma (EHE) has been controversial. [6] Controversy has existed in the literature also regarding whether Kimura disease and angiolymphoid hyperplasia with eosinophilia (ALHE) or ephiteloid hemangioma are the same entity. Some authors believe that Kimura disease represents a chronic, deeper form of ALHE; however, most papers distinguish the two on the basis of clinical and histopathologic characteristics. $[7,8]$ ALHE appears to represent an arteriovenous malformation with secondary inflammation. Kimura disease may represent a primary inflammatory process with secondary vascular proliferation. [9] There are problems in diagnosis in ephiteloid hemangioma, specially in cases with metaplastic bone formation like in our case. In those cases we should consider in differential diagnosis epithelioid hemangioendothelioma (EHE), epithelioid angiosarcoma (EA), and metastatic carcinoma. In oral cavity ephiteloid hemangioma may be presented with the infiltration of the bone and may show ulcerative changes with high rate of recurrence. There are 4 cases reported with location on the tongue, one reported case was reported as a diffuse intramuscular form of epitheloid hemangioma with ulcerative changes mimicking a malignant tumor with recurrence after surgery. [10]

Nielsen at all analyzed pathologic features of 50 epithelioid hemangiomas. The tumors arose in long tubular bones (40\%), short tubular bones of the distal lower extremity $(18 \%)$, flat bones $(18 \%)$, vertebrae (16\%), and small bones of the hands $(8 \%)$. Nine patients $(18 \%)$ had involvement of more than 1 bone. None of them was in maxillofacial region. They treated thirty-five patients with curettage, 13 patients had a local resection and 2 patients only had a biopsy. One patient had local lymph node involvement. Three patients were treated with surgery and radiation therapy. Follow-up information revealed that 4 patients experienced a local recurrence; and 1 patient developed limited involvement of a regional lymph node.[11]

Radiation as choice of the treatment after surgery can also be used either alone or in combination with surgery, particularly in nonoperable lesions or metastatic disease.

\section{Conclusion}


In our case, clinical behavior of the disease showed very aggressive behavior with recurrences which are in contradiction with our HP result. In a literature there are only a few cases of recurrence. When epithelioid hemangioma $(E H)$ of the bone is suspected, the differential diagnosis can include epithelioid hemangioendothelioma (EHE), epithelioid angiosarcoma (EA), and metastatic carcinoma. The importance of this case is that we must know to distinguishing $\mathrm{EH}$ from other malignant epithelioid vascular tumors as a result of differences in their management and clinical outcome.

\section{References}

1. Saraswathi, S. Nalin Kumar, P. Jayanthi, M. Umadevi, K. Ranganathan, and M. R. Srinivasan, "Epithelioid hemangioma of the alveolar mucosa," Journal of Oral and Maxillofacial Pathology, vol. 9, no. 1, pp. 21-23, 2005.

2. Al-Muharraqi $M^{1}$, Faqi MK, Uddin F, Ladak K, Darwish A., Angiolymphoid hyperplasia with eosinophilia (epithelioid hemangioma) of the face: An unusual presentation, Int J Surg Case Rep. $2011 ; 2(8): 258-60$. doi: 10.1016/j.ijscr.2011.03.007. Epub 2011 Sep 1.

3. Jessica M. Sin, ${ }^{1}$ Andrew H. Beck, ${ }^{2}$ Reetesh K. Pai, ${ }^{2}$ and Kathryn J. Stevens ${ }^{1}$, Multifocal Epithelioid Hemangioma with Reactive Bone Formation, ,ISRN Pathology, Volume 2011 (2011), Article ID 378940, 6 pages http://dx.doi.org/10.5402/2011/378940

4. Águida Cristina Gomes Henriques, Manuela Torres Andion Vidal, Clarissa Araújo Gurgel, Sanyra Lopes Dias Rocha, Braulio Carneiro Júnior, Roberto Almeida de Azevedo, Jean Nunes dos Santos, Epithelioid hemangioma in the oral mucosa, Srp Arh Celok Lek. 2016 SepOct;144(9-10):535-540

DOI: 10.2298/SARH1610535H

ПРИКАЗ

БОЛЕСНИКА / CASE REPORT UDC: 616.31006.31

5. Rajat Nangia, Abhiney Puri, Rakhi

Gupta, Sucheta Bansal, Amita Negi, Isha Chauhan, Epithelioid Hemangioma of Lingual Alveolar Mucosa: An Immunohistochemical Case Report, Case Reports in Medicine,Volume 2014 (2014), Article ID 436240, 5 pages, http://dx.doi.org/10.1155/2014/436240

6. Costantino Errani, MD, ${ }^{1,2}$ Lei Zhang, MD, ${ }^{2}$ David M. Panicek, MD, ${ }^{3}$ John H. Healey,
MD, ${ }^{1}$ and Cristina R. Antonescu, Epithelioid Hemangioma of Bone and Soft Tissue: $A$ Reappraisal of a Controversial Entity, , MD®2, Clin Orthop Relat Res. 2012 May; 470(5): 14981506.

7. Ramchandani PL, Sabesan T, Hussein $K$. Angiolymphoid hyperplasia with eosinophilia masquerading as Kimura disease. $\mathrm{Br} J$ Oral Maxillofac Surg. 2005 Jun. 43(3):24952. [Medline].

8. García Carretero R, Romero Brugera M, RebolloAparicio N, Vazquez-Gomez O. Eosinophilia and multiple lymphadenopathy: Kimura disease, a rare, but benign condition. BMJ Case Rep. 2016 Aug 31. 2016:[Medline].

9. García Carretero R, Romero Brugera M, RebolloAparicio N, Vazquez-Gomez O. Eosinophilia and multiple lymphadenopathy: Kimura disease, a rare, but benign condition. BMJ Case Rep. 2016 Aug 31. 2016:[Medline].

10. Tetsuo Shimoyama, Norio Horie, Fumio Ide, Epithelioid hemangioma of the tongue mimicking a malignancy, Journal of Oral and Maxillofacial Surgery, November 2000, Volume 58, Issue 11, Pages 1317-1319

11. Nielsen GP., Srivastava A, Kattapuram S, Deshpande V, O'Connell JX, Mangham CD, Rosenberg AE., Epithelioid hemangioma of bone revisited: a study of 50 cases, Am J Surg Pathol. 2009 Feb;33(2):270-7. doi: 10.1097/PAS.0b013e31817f6d51 